\title{
Desarrollo de políticas inclusivas en la educación superior
}

\author{
Development of Inclusive Policies in \\ Higher Education
}

\author{
Alicia Benet-Gil (D) https://orcid.org/0000-0002-6143-6292 \\ Universitat Jaume I, España, abenet@uji.es
}

\begin{abstract}
The diversity of university students and the emerging inclusive policies justify the intention to explore their applicability in the field of higher education. The aim of this study is to investigate the policies and regulations promoted by the university to guarantee access and permanence of students and also to find out how the university community perceives it. For this purpose, the case study has been used and interviews and focus groups with the rectoral team, university services, teachers and students have been carried out. Furthermore, the main documents of the institution has been analyzed. The main results show how the Universitat Jaume I is organized to address diversity and promotes actions aimed at this, however participants consider that some aspects of access and permanence should be improved. The conclusions point out the need to generate an inclusive policy that develops all services and regulations from an intercultural and inclusive approach.
\end{abstract}

Key words: policies, higher education, services, intercultural and inclusive approach.

Resumen: La diversidad del alumnado universitario y las políticas inclusivas emergentes justifican la intención de explorar su aplicabilidad en el ámbito de la educación superior. Este estudio pretende indagar en las políticas y normativas que promueve la Universidad Jaume I para garantizar el acceso y permanencia de los estudiantes y en cómo percibe esto la comunidad universitaria. Para ello se ha recurrido al estudio de caso y se han realizado entrevistas y grupos focales para el equipo rectoral, para los responsables y técnicos de los servicios que atienden a la diversidad del alumnado, los docentes y los estudiantes. También se ha efectuado un análisis de los principales documentos de la institución. Los resultados muestran cómo la Universitat Jaume I se organiza para atender la diversidad y fomenta acciones

Recepción: $15 / 08 / 18$

Aprobación: 02/05/19 dirigidas a ello, pero los participantes consideran que deben mejorarse algunos aspectos relativos al acceso y la permanencia. Como conclusión se apunta a la necesidad de generar una política inclusiva que englobe a todos los servicios y normativas desde un enfoque intercultural e inclusivo.

Palabras clave: políticas, educación superior, servicios, modelo intercultural e inclusivo. 


\section{Introducción}

La inclusión de las personas es un principio que exige la capacidad de mirar la diversidad desde el respeto a las diferencias y garantizando la participación activa de todos en los procesos educativos. Ese principio se traslada a la educación superior en el intento de desarrollar un modelo que abarque simultáneamente los conceptos de igualdad, equidad, accesibilidad y excelencia educativa.

El modelo intercultural inclusivo ha sido desarrollado en el ámbito escolar para hacer efectivo el derecho a la no discriminación y a la participación de todos los estudiantes, independientemente de su condición social o cultural, de su género o de sus características personales. A pesar de que en España la educación intercultural y la educación inclusiva se han llevado a cabo de manera independiente, consideramos que ambos enfoques comparten el compromiso de transformar la educación para dirigirse hacia valores y actitudes democráticos de igualdad, respeto, autonomía y solidaridad (Moliner et al., 2011; Sales et al., 2012).

Por tanto, podemos hablar de un modelo intercultural inclusivo "que propicia el enriquecimiento cultural de los ciudadanos, partiendo del reconocimiento y del respecto a la diversidad, a través del intercambio y el diálogo, en la participación activa y crítica para el desarrollo de una sociedad democrática basada en la igualdad, la justicia y la solidaridad" (Sales y García, 1997: 46). Este modelo es el que debe orientar las políticas educativas también en la educación superior.

\section{Educación superior inclusiva en el contexto latinoamericano y europeo}

La política internacional y europea destaca la inclusión y el acceso a la educación como un derecho humano. La pregunta no es si debe haber inclusión en la educación en general y en la educación superior específicamente, sino qué mecanismos ponemos en marcha para lograrlo de forma efectiva y exitosa.

La legislación es una parte vital del desarrollo de un modelo inclusivo, pues articula los principios y derechos necesarios para crear un marco y detectar las barreras que impiden el acceso y la permanencia en la educación. Asimismo, la legislación es fundamental para estructurar las prácticas inclusivas que garantizan una educación para todas las personas (Unesco, 2017). La política educativa puede influir en las prácticas inclusivas, definiendo las formas de enseñanza, apoyo y liderazgo que constituyen la base de una educación de calidad (Unesco, 2015). 
La educación superior en el contexto latinoamericano se organiza de manera compleja y diversa. En un estudio realizado por Sverdlick et al. (2005) en cinco países de América Latina, se pone de manifiesto que las universidades latinoamericanas han sufrido muchas reformas, aunque estas no han significado una modificación sustancial de las características elitistas de las mismas. Actualmente, las diferentes estrategias para la inclusión con el apoyo de acciones y políticas públicas han dado lugar a cambios sustanciales en la matrícula universitaria y en la composición de sus diversas poblaciones.

Las instituciones de educación superior de algunos países realizan esfuerzos de acción positiva para ampliar el acceso de poblaciones poco favorecidas o vulnerables a la educación superior, pero las desigualdades de acceso/permanencia persisten, constituyéndose en un serio desafío para la elaboración e implementación de políticas de inclusión a nivel nacional e institucional.

En este marco encontramos algunos ejemplos de proyectos inclusivos como el MISEAL (2012-2014) (Rifa, 2014), desarrollado por 12 instituciones de educación superior latinoamericanas y cuatro europeas, cuyo objetivo principal es promover procesos de inclusión social y equidad en instituciones de educación superior en América Latina, mediante el desarrollo de medidas para mejorar los mecanismos de acceso, permanencia y movilidad, la formación de expertos en inclusión y equidad, y la articulación de una red de colaboración entre universidades europeas y latinoamericanas.

Las actividades no están orientadas a grupos tradicionalmente excluidos como: "minorías" étnicas o raciales, personas económicamente desfavorecidas, personas con discapacidad, personas discriminadas por cuestiones de género, sexualidad y edad.

En Europa, la conferencia Access4All promovida por la Comisión Europea (European Commission, 2017) tuvo como objetivo fomentar la evaluación de políticas institucionales y las buenas prácticas desarrolladas por organizaciones de educación superior en Europa, con el fin de impulsar la inclusión educativa y social de grupos minoritarios, así como de alumnos con alguna necesidad.

Por otro lado, la Unesco (2017: 13) pone de manifiesto los requisitos que debe recoger la política educativa para garantizar la inclusión:

- Valorar la presencia, la participación y los logros de todos los estudiantes, independientemente de sus contextos y características personales.

- Reconocer los beneficios de la diversidad de los y las estudiantes y cómo vivir con las diferencias y aprender de ellas. 
- Recopilar, cotejar y evaluar evidencias sobre las barreras al acceso, a la participación y a los logros, prestando especial atención a los y las estudiantes que pueden estar más expuestos al fracaso, la marginación o la exclusión.

- Aplicar cambios de forma eficaz y supervisar su impacto, reconociendo que el logro de la inclusión y de la equidad en la educación es un proceso continuo, más que un esfuerzo único.

Según Escudero (2006), la política es la plataforma en la cual se sustenta un modelo educativo que se concreta en las prácticas. Por tanto, es una decisión eminentemente política la que permite una mejora en la educación. También Aguado et al. (2006) señalan que la educación es intrínsecamente un asunto político y, por ello, plantean tres niveles de política educativa:

1. Proyectos educativos en el ámbito internacional, europeo o local, que se expresan en declaraciones, recomendaciones o documentos de trabajo.

2. Las leyes y las normas explícitas que tratan de organizar el sistema educativo.

3. La práctica real que se refiere a las medidas concretas que los agentes educativos desarrollan para aplicar las leyes y normas.

Diseñar una política desde el enfoque intercultural inclusivo requiere una reflexión acerca de las barreras que impiden la participación en términos económicos, políticos, sociales, físicos, culturales o lingüísticos. Así pues, las políticas inclusivas en la educación superior deben caracterizarse por (Ministerio de Educación de Colombia, 2013):

- Identificar las barreras institucionales que impiden el ejercicio efectivo de una educación inclusiva.

- Hacer énfasis en aquellos estudiantes que pueden estar en riesgo de exclusión.

- Promover una participación activa de toda la comunidad académica, especialmente de los alumnos.

- Proponer acciones de acompañamiento que permitan garantizar la permanencia de los estudiantes.

Al tomar como referencia el Index for Inclusion (Booth y Ainscow, 2000) y la Guía CEIN (Sales et al., 2010), encontramos una serie de elementos indispensables para asegurar la inclusión de los estudiantes en la universidad. La revisión de la literatura nos remite a dos momentos: el acceso y la permanencia (véase Figura 1). ${ }^{1}$

1 Las figuras y las tablas se encuentran en el Anexo, al final del presente artículo (Nota del editor). 
A partir de la idea de que tanto las prácticas como las culturas de las instituciones de educación superior están mediatizadas por las políticas organizativas, en este estudio pretendemos abordar el desarrollo de las políticas inclusivas en un contexto concreto, el de la universidad española Universitat Jaume I (UJI). Se trata de analizar las políticas de organización institucional que, en su vertiente normativa y estructural, y articuladas por los servicios responsables, intentan avanzar en la construcción de una universidad más inclusiva.

\section{Objetivo y preguntas de investigación}

Esta investigación se encuadra dentro de un trabajo más amplio que busca identificar el estado actual de las culturas, políticas y prácticas de la comunidad educativa de la UJI, en el marco de la universidad intercultural inclusiva. Pretendemos indagar en las políticas, normativas y organización de la universidad, que procuran garantizar el acceso y permanencia de todos los estudiantes. Para ello, nos planteamos tres preguntas de investigación:

- ¿Cómo se estructura la universidad para dar respuesta a la diversidad y qué opina la comunidad educativa acerca de ello?

- ¿Qué políticas, normativas y planes permiten el acceso a la universidad y cómo lo perciben los participantes?

- ¿Qué políticas, normativas y planes promueve la universidad para garantizar la permanencia de sus estudiantes y qué creencias tienen los participantes acerca de estas?

\section{Metodología}

Este trabajo se enmarca dentro de la investigación cualitativa y la metodología empleada en el estudio de casos. Tal y como apunta Pérez (1994: 85), podríamos definirlo como "una descripción intensiva, holística, y un análisis de una entidad singular, un fenómeno o unidad social". Se trata de un estudio de caso centrado en la UJI, cuya finalidad es conocer su particularidad, complejidad, singularidad y exclusividad (Stake, 1998; Simons, 2011) en cuanto al desarrollo de políticas inclusivas.

\section{Contexto}

La UJI es una universidad pública de Castellón (España), creada en 1991 y que actualmente acoge alrededor de 15.000 estudiantes. Se organiza en 
cuatro facultades (Ciencias Humanas y Sociales, Salud, Ciencias Jurídicas y Económicas, y Escuela Superior de Tecnologías y Ciencias Experimentales) que ofertan un total de 32 estudios de grado, 47 másteres universitarios y 20 programas de doctorado. La UJI integra una serie de servicios relacionados con la atención a la diversidad como son: Unidad de Apoyo Educativo (USE), Servicio Gestión a la Docencia y Estudiantes (SGDE), Unidad de Igualdad (UI), Servicio de Lenguas y Terminología (SLT), Oficina de Relaciones Internacionales (ORI), Oficina Cooperación al Desarrollo y Solidaridad (OCDS), Oficina de Prevención y Gestión Medioambiental (OPGM) y el grupo de investigación Salusex (véase Tabla 1).

\section{Participantes}

Para elegir a los participantes del presente estudio se contactó con los responsables y técnicos de los servicios relacionados con la atención a la diversidad del alumnado, mencionados en el párrafo anterior. Respecto a la selección del profesorado, se realizó con la colaboración de dichos servicios, cuyos responsables nos propusieron una serie de docentes que se encontraban sensibilizados con la atención a la diversidad. De todo el listado sugerido por las diferentes unidades, se intentó representar a las distintas facultades, así como la igualdad representativa de hombres y mujeres.

En cuanto a la elección de estudiantes, el criterio se basó en tener la mayor diversidad posible: social, cultural, de género, funcional, económica, edad, carrera, años en la universidad, etc. Para ello, la mediación se efectuó a través de los diferentes servicios. Se seleccionó a todos los alumnos que contestaron y aceptaron colaborar en el estudio. Respecto al equipo rectoral, participó un $80 \%$ de sus integrantes además del rector. En total fueron 40 participantes; la Tabla 2 reúne los datos.

\section{Técnicas e instrumentos de recogida de información}

Los instrumentos de recogida de información se han elaborado ad hoc, a partir de los indicadores de las guías de autoevaluación para la escuela intercultural inclusiva revisados, que fueron: The Index for Inclusion (Booth y Ainscow, 2000) y Guía CEIN (Sales et al., 2010). Con base en los ítems de la dimensión "políticas", diseñamos un protocolo para el análisis documental, el guión para los grupos focales y los guiones de las entrevistas semiestructuradas. 
El grupo focal, según Cerón (2006: 279), “es especialmente útil para el estudio de racionalidades o lógicas de acción en un colectivo determinado y respecto a un campo práctico determinado, porque permite la comprensión de la acción, al interpretarla desde la perspectiva del actor que la realiza". Se organizó un total de cinco grupos focales: dos para el profesorado y tres para el alumnado. El guión es un protocolo que consta de dos apartados: a) datos contextuales y de los informantes; y b) tres preguntas generales.

La entrevista semiestructurada nos sirvió para descubrir las creencias, pensamientos y valores de las personas participantes. La elección de este instrumento, a diferencia de los grupos focales, obedeció a que nos interesaba conocer en profundidad cómo se organiza y estructura la universidad. Se realizó un total de 20 entrevistas: 11 a personal de los servicios, 1 a un docente y 8 a los vicerrectores y al rector. El guión de entrevista semiestructurada consta de tres apartados: a) datos contextuales; b) datos del informante; y c) preguntas agrupadas sobre los indicadores de políticas.

También se hizo un análisis de los principales documentos de la institución, para conocer su ideario, organización y funcionamiento. Estos documentos se pueden ver en la Tabla 3.

\section{Análisis de datos y codificación}

Se llevó a cabo un análisis temático de los datos obtenidos. Se trata de un método que permite examinar en detalle y proporcionar patrones o temas a partir de una cuidadosa lectura de los datos recogidos, para comprender e interpretar el fenómeno de estudio (Braun y Clarke, 2006). Las entrevistas, grupos focales y los documentos institucionales se codificaron y categorizaron, identificando partes de texto con un tema y relacionándolo con un código, que era una referencia abreviada de una idea temática (Gibbs, 2007).

El análisis de datos ha sido un temático mixto: deductivo a partir de dos temas centrales: el acceso y la permanencia; e inductivo, con base en las nuevas categorías emergentes que dan cuenta de la configuración temática de los resultados. Los datos fueron analizados con el programa de análisis de datos cualitativos Atlas.ti (véase Tabla 4).

\section{Resultados}

La primera pregunta de investigación pretendía indagar sobre cómo se estructura la Universidad Jaume I para dar respuesta a la diversidad y qué percepción tiene la comunidad universitaria acerca de ello (véase Figura 2). 
Los datos apuntan a que la universidad delega en los servicios la gestión técnica, económica y administrativa, el apoyo, la asistencia y el asesoramiento de toda la comunidad universitaria. Respecto a la atención a la diversidad, la universidad impulsa una serie de planes y normativas que impulsan las oficinas y servicios que participan en la investigación.

La colaboración es considerada un aspecto fundamental, y en la UJI es un valor que aparece de manera explícita en los estatutos y en el código ético. Respecto a la colaboración entre los servicios, los encargados aseguran que se coordinan en función de las necesidades, pero que no existe un organismo o convenio que coordine esa relación.

Entre las actuaciones que se realizan de forma colaborativa, podemos encontrar la elaboración conjunta del protocolo de acoso entre Salusex y la Unidad de Igualdad; el trabajo entre el Plan de Atención a la Diversidad y el centro de salud en la adaptación del espacio de trabajo-estudio, las tareas entre el Servicio de Actividades Socioculturales y el de Lenguas y Terminología en la programación de actividades que promueven el valenciano, etc. Por otro lado, algunos de los servicios forman redes de trabajo para compartir experiencias; un ejemplo es la Red de Servicios de Atención a Personas con Discapacidad en las Universidades españolas (SAPDU), conformada por los servicios de apoyo a las personas con discapacidad de las universidades españolas, actualmente presidida por el equipo de la UJI.

Sin embargo, los participantes tienen opiniones encontradas respecto a esta colaboración. Mientras algunos responsables de los servicios afirman abogar por una cultura colaborativa, los estudiantes perciben que los servicios funcionan de manera independiente.

Por las características del servicio estamos en contacto con otros, tenemos una tarea muy interdisciplinar (E_S_ORI).

Una Unidad de Atención a la Diversidad no puede funcionar independientemente, debe relacionarse con la ORI, la Oficina de Prácticas, el profesorado, con todos (E_ VR7).

Las oficinas funcionan como compartimientos estancos, entre ellos no saben, te van mandando de un lugar a otro como pelotas de tenis (GF1_E).

La propuesta de mejora de los participantes en cuanto a la colaboración es realizar un plan integral de inclusión con todos los servicios:

Yo creo que interesa hacer un plan bien sostenible para que la integración, tanto por motivos físicos como culturales, que suelen ser los más graves, funcione (GF2_D6).

Hay veces que las acciones no terminan de estar conectadas la una con la otra y deberían de estar dentro de lo que es una política general (E_R). 
Por otra parte, existe una preocupación compartida por todos los participantes respecto a la política lingüística. Al tratarse de una universidad multilingüe, la comunidad universitaria puede encontrar las tres lenguas (valenciano, castellano e inglés) en la docencia, en la administración o en la investigación.

La gente que se matricula en nuestra universidad debe de ser consciente que encontrará docencia en valenciano, inglés y castellano (E_S_SLT).

La política lingüística no es compartida por toda la comunidad universitaria y eso genera tensión. Los docentes lamentan las actitudes de algunos estudiantes respecto al uso del valenciano en el aula y consideran que la universidad debe dejar más claro la política que se sigue.

En tanto, la diversidad afectivo-sexual es un tema pendiente. Actualmente la atención a estudiantes que necesiten apoyo o asesoramiento en estas cuestiones son atendidos por el grupo de investigación SALUSEX, pero este no forma parte de la estructura oficinas y servicios de la universidad, La UJI está intentando resolver cómo trabajar esta cuestión e incorporarla en su estructura.

Estamos intentando ver cómo enlazamos la diversidad afectivo-sexual y cómo incorporarla dentro de la estructura y la forma de trabajar (E_VR3).

Otro aspecto interesante es cómo la inclusión es tratada en los planes de estudio. Los participantes abogan por la necesidad de promover planes mucho más interdisciplinares y proponen que:

La discapacidad se debe trabajar desde todos los ámbitos y contemplarlo dentro de los planes de estudio (E_S_UDD).

Meter actividades dentro de nuestras asignaturas que conlleven esa parte de inclusión (GF1_D13).

Desde los vicerrectorados existe voluntad política en este sentido y consideran que:

El tema de la inclusión, del respeto cultural, de la integración, debe impregnar nuestros planes de estudio (E_R).

Sin embargo, parece que aún queda camino por recorrer, pues se afirma que:

Se está intentando incorporar asignaturas de igualdad, pero tenemos dificultades que vienen de la Agencia Nacional, no es nada fácil incorporarlo (E_VR6).

La segunda pregunta de investigación se refiere a las políticas y normativas de la universidad sobre el acceso del estudiantado y a la percepción de los participantes respecto a estos mecanismos (véase Figura 3). 
El acceso a la universidad puede realizarse a través de diferentes vías: a) Bachillerato, b) Formación Profesional, c) Pruebas para mayores de 25, 40 o 45 años, d) Estudios parciales, y e) Estudiantes internacionales.

La UJI cuenta con un programa de Orientación Preuniversitaria que facilita la transición de la secundaria a la universidad. Dentro de este programa encontramos diferentes acciones como: visitas a la institución, promoción de alumnado de alto rendimiento, incentivación de las vocaciones científicas, técnicas y humanísticas, captación de alumnado internacional y coordinación académica con los centros educativos preuniversitarios.

Por otro lado, uno de los recursos respecto al acceso a nivel informativo es el Sistema de Información Académica, donde se recogen los datos de la oferta de estudios oficiales, asignaturas y guías docentes, sistemas de evaluación, profesorado, horarios y aulas, e información sociolingüística de las materias que se van a cursar.

También se llevan a cabo Jornadas de Acogida y el Programa de Acción Tutorial (tutoría entre iguales), para facilitar la adaptación y la integración del estudiantado de nuevo ingreso, dando a conocer el funcionamiento de la universidad y los servicios que ofrece. En cuanto a los estudiantes internacionales, se realiza una jornada, donde se hacen visitas guiadas, se dan consejos de seguridad y normas de convivencia, y se explica la oferta de los cursos de lenguas.

Respecto al ingreso, los entrevistados señalan que hay recursos como las becas o el apoyo de la Unidad de Discapacidad y Diversidad (UDD) y de la Oficina de Relaciones Internacionales (ORI) que ayudan a la incorporación en la universidad.

La ORI funciona muy bien en conjunto con la oficina del máster para todo el proceso previo de llegada (GF2_E8).

En selectividad todos los años tenemos a una persona que se ha roto el brazo, o ha tenido un accidente, y la UDD pone a su servicio una persona que le ayude y esta persona va a un aula especial para poder hacer la selectividad (GF2_D6).

También se pone de manifiesto la importancia de las plazas para promover la incorporación de mujeres a grados masculinizados o de las plazas para personas con discapacidad para realizar estudios de grado y máster.

Los estudios de doctorado son los únicos que quedan a nivel estatal que no tienen tasa reservada de plaza para la discapacidad. Yo creo que es un aspecto que se corregirá en los próximos años (E_VR4).

Sin embargo, los entrevistados hacen referencia a una serie de barreras que pueden dificultar el acceso. Una de ellas alude al acceso para personas mayores de 25 años: 
Mi 7 en selectividad de hace 20 años no es el 7 de ahora, en ese momento esa nota me garantizaba entrar y ahora he entrado en Psicología, de casualidad (GF2_E7).

Al respecto, como propuesta de mejora, los participantes apuntan a la necesidad de ampliar los colectivos entre los que se hace difusión y no solo incorporar a los que provienen de los centros de educación secundaria, sino también a la formación profesional o a la población adulta.

La información sobre la universidad se hace en los centros de secundaria, se da alguna información en la Educación Secundaria Obligatoria, pero, evidentemente, donde más se hace es en Bachillerato, y no todos llegan a Bachillerato (E_VR2).

También mejorar la orientación vocacional para elegir carrera, ya que muchos estudiantes tienen expectativas erróneas.

Tenemos problemas en cómo arbitrar la orientación adecuada a los alumnos con ciertos hándicaps para que no solo entren a las carreras por su gusto o motivación, sino porque... Ahí sí que veo que algo habría que hacer, pero es un problema de difícil solución (E_S_USE2).

Otra de las problemáticas a las que se apunta es la falta de competencia lingüística de algunos estudiantes. En ese sentido, se apunta a la necesidad de solicitar requisitos lingüísticos y realizar un curso de inmersión para afianzar el castellano y acercarlos a la cultura valenciana.

Yo creo que a los Erasmus deberían hacerles un examen y exigirles un mínimo, y si no llegan hacerles clases alternativas. Creo que hay que ponerles un poco las pilas. Es mejor perder un semestre para aprender castellano bien que no perder todo el año (E_D8).

Yo creo que habría estado bien que nos explicaran la cultura valenciana (GF2_E9).

Probablemente en un par de años deberemos pedir el español porque hay algunos profesores que tienen Erasmus que se están quejando (E_VR3).

La última pregunta de investigación pretende conocer las políticas que promueve la universidad para garantizar la permanencia de los estudiantes, sea cual sea su situación, y qué opinión tiene la comunidad universitaria acerca de estas.

En los estatutos de la Universidad Jaume I se reconoce la obligación de facilitar al alumnado la continuación de sus estudios. Esto se realiza mediante diferentes mecanismos como: la accesibilidad, las normativas, la orientación, la participación y los apoyos (véase Figura 4).

La accesibilidad es un factor que se relaciona tanto en el acceso a la universidad como en la permanencia de los estudiantes. La percepción del equipo rectoral es que la universidad es accesible y se realizan acciones encaminadas a ello. 
Yo creo que si hay algo de lo nos enorgullecemos es de tener un campus con un grado de accesibilidad muy alto (E_R).

Nuestra universidad, al construirse dentro de este siglo, los edificios y todo eso hace que sea mucho más accesible. Todo lo que marca la legalidad se sigue (E_VR5).

Yo considero que es accesible, no solo desde el punto de vista físico, yo diría que puede considerarse una universidad inclusiva (E_VR7).

Hay tres tipos de accesibilidad: física, comunicativa y económica. Respecto a la primera, la UJI es un campus único e integrado. Al ser una universidad de reciente creación, se construyó bajo normativas de accesibilidad. Se trata de un aspecto que no solo está desarrollándose en la práctica, sino que se recoge en los estatutos, en el modelo educativo y en el código ético. Cabe destacar que la universidad tiene algunos premios de reconocimiento por su accesibilidad; el equipo de gobierno considera que se han hecho muchos esfuerzos para facilitar la movilidad por la universidad, sobre todo, para las personas con discapacidad, aspecto bien valorado por la comunidad universitaria.

La idea es que toda construcción que se haga esté preparada para que la gente que tenga problemas pueda acceder a la universidad (E_VR5).

La primera vez que utilicé la tarjeta azul para aparcar en las zonas reservadas me entró mucho sentimiento. Pero bueno, a veces he aparcado al campo de fútbol y cuando llegaba a clase ya no tenía ganas de nada (GF2_E7).

En este sentido, otro aspecto considerado importante es la señalización de la universidad. El vicerrectorado expone que se ha trabajado en esta parte, aunque hay cuestiones por mejorar.

En lo que sí que hemos trabajado explícitamente es en la señalización para la gente que entra a la universidad en un vehículo, porque normalmente la gente se perdía. Hay determinadas zonas que son complicadas. Ahí lo que procuramos es, por un lado, que estén bien señalizadas y por otro, atender a todas las demandas para mejorar (E_VR5).

Yo de aquí no sé salir si no pregunto la mujer que limpia, me puedes tener aquí una hora dando vueltas (GF2_E7).

Por su parte, el Plan de Atención a la Diversidad (PAD), junto con la Oficina de Prevención y Gestión Medioambiental, realiza diferentes acciones para garantizar la accesibilidad de la comunidad universitaria, como: adaptaciones del lugar de estudio, del aula o de los horarios; promover espacios de descanso en las facultades o dar apoyo en el desplazamiento por el campus.

Por otro lado, el acceso a la información es un aspecto imprescindible en la permanencia en la universidad. El Real Decreto 1791/2010 del Estatuto del Estudiante universitario pone de manifiesto la necesidad de que 
los alumnos dispongan de la adecuada información oficial sobre el horizonte temporal en el cual se concreta la permanencia en sus estudios.

En este sentido, el lenguaje es clave, y la política lingüística juega un papel muy importante en el acceso comunicativo. La universidad incorpora en sus planes y normativas el concepto de seguridad lingüística para referirse a la transparencia informativa de los usos lingüísticos docentes; es decir, hacer pública la información sobre la lengua vehicular de la docencia en el Sistema de Información Académica comentado anteriormente. Esto supone el compromiso de respetar la lengua vehicular declarada como parte del contrato del estudiante con la universidad y que debe conocerse con antelación.

Respecto a ello, la comunidad universitaria tiene opiniones contrapuestas. El equipo rectoral y de los servicios consideran que existen muchos canales de comunicación para hacer accesible la información.

Recientemente hemos hecho una mejora sustancial de la web y se ha creado un apartado específico para estudiantes antes de llegar a la universidad, otra cuando ya están y otra para estudiantes egresados. Creo que se han hecho esfuerzos para dar coherencia y unificar la información (E_VR6).

Nosotros trabajamos mucho con el servicio de comunicaciones para que la web sea accesible (E_S_USE1).

Sin embargo, los estudiantes consideran que la información que existe en estos canales no siempre es correcta y varia en función del idioma:

Cuando son concursos, becas... todos, todos, son en valenciano y a veces ni siquiera en los dos idiomas (GF2_E9).

La priorización es la web internacional y las guías docentes. Hay cosas que no es necesario traducir (E_SLT).

Por otro lado, los estudiantes se quejan de que la información no siempre está en los tres idiomas y cuando está no siempre es la misma, cuestión que corrobora el Servicio de Lenguas y Terminología.

A veces me encuentro que el contenido en valenciano, en castellano o inglés no coincide (GF1_E2).

Es muy importante saber que la web no es responsabilidad nuestra. Nosotros mismos encontramos traducciones en inglés o valenciano que... Cada servicio, unidad y departamento es responsable de su web. Muchos lo que hacen es utilizar el Google Traductor y... Nosotros estamos aquí para ayudar, pero la inmediatez de la web... Es un problema que está encima de la menos y que de algún modo hay que abordar (E_SLT).

Como propuesta se apunta a la necesidad de presentar la información en los tres idiomas, para que los estudiantes de otros países puedan acceder a ella de manera completa. 
Pasarlo al castellano o al inglés sería lo ideal (GF1_E2).

Respecto a la accesibilidad económica, los estudiantes cuentan con diferentes becas y ayudas para costear la matrícula, el alojamiento u otros gastos. Hay tres tipos de becas según quién las financia: el Ministerio de Educación, la Consellería o la propia universidad. Además, hay otro tipo de ayudas para el comedor, la asistencia a cursos, jornadas o congresos, etc. Dentro de estas, merece la pena destacar las ayudas para situaciones sobrevenidas para estudiantes que se encuentren en circunstancias como la pérdida de empleo, la incapacidad o la muerte de algún miembro de la familia que conlleve la disminución de ingresos. Por otro lado, existen otro tipo de becas dirigidas a estudiantes que provienen de países empobrecidos.

El equipo rectoral considera que hay muchos mecanismos para que cualquier alumno pueda realizar sus estudios sin que la economía sea un problema.

Yo te diría que, si hoy en día combinamos las becas del Ministerio, Generalitat y las de la propia universidad, que no se queda ningún estudiante sin estudiar por una cuestión puramente económica (E_VR6).

La beca que yo estoy disfrutando está enfocada a gente de Asia y África, y Latinoamérica.

O sea, se está buscando estudiantes de fuera de España y se está intentando ayudarlos económicamente (GF1_E3).

En relación con la permanencia, hay una serie de normativas que inciden directamente en el alumnado: de Permanencia, de Compensación Curricular y de Evaluación. Cabe destacar que en ellas se tiene en especial consideración a los estudiantes con discapacidad y, en los últimos años, a los estudiantes con necesidades educativas específicas. Un ejemplo de ello es que existe la obligación de hacer adaptaciones, ya sea a nivel curricular o de evaluación.

La participación es otro de los valores que promueve la universidad y se sustenta en el voluntariado y el asociacionismo. La UJI tiene un programa propio de voluntariado, y la gente de los servicios lo utilizan para emprender acciones de sensibilización, acompañamiento y apoyo entre alumnos. El asociacionismo es otro aspecto clave, la universidad tiene una normativa propia y actualmente figuran 29 entidades inscritas de diversas temáticas.

Sin embargo, de estas 29 solo 6 están gestionadas por estudiantes. Las creencias de los entrevistados son dispares: mientras el equipo rectoral considera que promueven la participación de los estudiantes; los alumnos opinan que falta más representación estudiantil y más visibilidad de algunos colectivos.

Hemos transformado el asociacionismo. Ahora están mucho más comprometidos, hemos dado protagonismo a las asociaciones, hemos hecho un programa de ayudas (E_VR7). 
Yo es que veo que esta universidad adolece de falta de asociacionismo. ¿Dónde contactas con los estudiantes? Hay mucho grupo de trabajo, muchos docentes y departamentos, pero, ¿de estudiantes? No veo yo (GF3_E13).

Yo creo que falta algún tipo de asociación de LGTBI porque no la hay. Estaría bien que hubiera un departamento formado por estudiantes (GF3_E12).

Respecto al voluntariado, los responsables y técnicos de los servicios valoran muy positivamente el compromiso de los estudiantes.

Estamos súper contentos con la predisposición, ahí no tenemos ningún problema, lo contrario. A veces necesitamos tres personas y se presentan 20 (E_S_USE3).

La orientación, junto con el apoyo, son los pilares más fuertes dentro de la permanencia de los estudiantes. Por un lado, los servicios ofrecen asesoría en movilidad, idiomas, conciliación familiar, necesidades educativas específicas, aspectos académicos y cómo afrontar una situación de acoso. Por otro lado, se realizan orientaciones al profesorado mediante formación. Esta gestión la realiza la Unidad de Formación e Innovación Educativa. Actualmente, la formación en atención a la diversidad tiene poca incidencia y se efectúa bajo demanda del profesorado. Se puede decir que esta se traduce en las reuniones de asesoramiento, orientación y seguimiento que se hacen desde el Plan de Atención a la Diversidad.

Los técnicos de los servicios comentan que los docentes, en general, buscan en los cursos la respuesta a sus necesidades y por eso, muchas veces, algunos no tienen demanda.

La mayoría de gente busca lo aplicado, recetas. Este tipo de cursos de atención a la diversidad es complicado que tengan éxito, hay que venderlo como muy muy práctico (E_S_USE2).

La política formativase dirige a los grupos de innovación educativa y a los intereses de estos; por lo tanto, no hay ninguna formación específica en atención a la diversidad que se dirija a todo el profesorado.

Más que iniciativas de la propia universidad para el tema de la diversidad veo más factible que grupos de profesores se preocupen por mejorar directamente y que, según sus necesidades, salga la formación que les haría falta (E_S_USE2).

$\mathrm{Al}$ respecto, cabe destacar la opinión del rector:

Hay docentes que respecto a una situación en el aula no han sabido reaccionar o se han quejado de no tener la formación necesaria, lo cual, no sé si es culpa de él, que se ha preocupado poco por formarse, o hace falta algo más activo desde la universidad para dar esa formación (E-R).

Los participantes consideran que, para avanzar a una universidad inclusiva, es necesario tener formación en atención a la diversidad, aunque aún no 
tienen claro cuál es el mejor modo de abordarla. Por otro lado, el profesorado está de acuerdo con esta necesidad, pero se queja de la carga burocrática que existe en la institución y de la poca valoración que se da a la docencia.

No hay una formación de atención a la diversidad que debería ir de la mano de un curso de igualdad (E_S_USE2).

La internacionalización es un tema que es necesario reforzar en la formación del profesorado (E_VR2).

No sé, yo sería muy cauto en estas cosas, cómo se lo vendes a la gente para que vea una necesidad real. Sobre el papel te diría que sí, que merece la pena, pero en la práctica me he dado cuenta que de dónde sacamos el tiempo y a costa de qué (GF2_D6).

Por último, cobran gran relevancia los apoyos, entre ellos hallamos las becas y los programas específicos. Aparte de las becas para la matrícula o el alojamiento, la universidad tiene una bolsa de alojamiento pública que facilita la búsqueda de piso, dispone de una residencia universitaria dentro del campus y el Programa "Pisos Solidarios", donde los estudiantes tienen gratuidad de alquiler a cambio de unas horas semanales de participación en actividades para mejorar un barrio de la ciudad.

Dentro de los programas específicos encontramos uno de los más fuertes y que más apoyo ofrece: el Plan de Atención a la Diversidad (PAD), dirigido a estudiantes y docentes con discapacidad o Necesidades Educativas Especiales y gestionado por la UDD. Este brinda ayuda desde las pruebas de acceso a la universidad, a través de aulas de incidencia, y durante toda su trayectoria académica.

Cuando un estudiante se matrícula en la universidad por primera vez y advierte que dispone de un certificado de discapacidad o cualquier otra circunstancia, la unidad se pone directamente en contacto para ofrecerle apoyo y asesoramiento. Las principales funciones de esta unidad son: asesoramiento psicopedagógico (seguimiento académico y mediación con el profesorado, gestión de exámenes, apoyo para realizar las prácticas...), adaptación del lugar de estudio/aula, información sobre becas y ayudas específicas, apoyo en: gestiones académicas, necesidad de espacios de descanso, desplazamientos por el campus o ayuda de compañeros voluntarios.

La UDD se posiciona como uno de los principales apoyos en la universidad; todos los participantes del estudio hacen una valoración muy positiva del servicio y manifiestan que es de gran ayuda no solo para estudiantes, sino para los docentes.

Mi relación con la universidad empieza con esta unidad y ellas me orientan en todo lo que haga falta (GF2_E7). 
Tenemos una persona, un servicio, no estamos perdidos. Si no tuviéramos eso dirías, ¿ahora qué hago? Porque siempre están ahí, al teléfono... (GF2_D7).

Los docentes resaltan como aspecto positivo, el que la unidad les de información previa sobre estudiantes que requieren de algún tipo de adaptación o consideración; además de las orientaciones pedagógicas que reciben por parte de las responsables para dar una respuesta adecuada a los estudiantes.

Una cosa que me fascina, y creo que es un rasgo que nos diferencia de otras universidades que nos han copiado y nos han copiado para bien, es el tema de la UDD. Cuando llega un estudiante de un instituto y han detectado una necesidad especial, tú tienes esa información previa (GF2_D6).

Cuando estuve embarazada me dijeron que había una sala de descanso por si quería ir, incluso cuando tuviera al bebé por si quisiera darle de mamar (GF2_E8).

Me dijeron si quería reducir las horas de clase y les dije que no, que prefería que me quitaran horas de investigación, porque no puedo estar mucho tiempo delante del ordenador (E_D8).

Hace un tiempo tuvimos un caso puntual de un estudiante y las responsables del servicio vinieron a decirnos a los profesores cuatro consejos sobre cómo utilizar el espacio y el tiempo con ese estudiante (GF2_D6).

Por su parte, el personal de la Unidad de Discapacidad y Diversidad propone una mayor coordinación con el vicedecanato.

Lo ideal sería hacer reuniones de coordinación desde la titulación utilizando la figura del vicedecano y así serviría como referencia para el profesorado. Porque nosotros, por mucho que sepamos y estemos en contacto con los estudiantes, no dejamos de ser un personal de administración y servicios y, a veces, el profesorado no siempre te mira bien (E_S_USE1).

Siguiendo con los programas específicos encontramos los que se refieren a los idiomas y a la internacionalización. La universidad invierte mucho en el aprendizaje de idiomas a través del voluntariado, de la formación, asesoramiento, de recursos o de incentivos y ayudas, ya sea para enseñar idiomas o para fomentar la docencia en valenciano o inglés. Sin embargo, existen opiniones encontradas acerca de la oferta de cursos de español y valenciano: el Servicio de Lenguas y Terminología ha manifestado su interés por difundirlos y promoverlos; pero los estudiantes y docentes desconocen dicha oferta o tienen quejas sobre los horarios y el acceso.

Los cursos de español tienen un precio estipulado y van dentro del convenio Erasmus y es casi obligatorio inscribirse. Los de valenciano son gratuitos para el nivel A2. Lo que hacemos es abrir las puertas de nuestra lengua a la gente que viene de fuera (E_S_SLT). 
Me he encontrado alumnos que han ido a matricularse y resulta que están llenos. Al final se han ido de aquí sin aprender valenciano ni mejorar el español, porque han cogido todas las asignaturas en inglés (GF1_D1).

Casi todos los cursos de valenciano son de pago, yo estoy en una situación desesperada por temas económicos (GF2_E9).

La internacionalización va de la mano de las políticas lingüísticas, y el apoyo se brinda mediante la formación o los programas específicos.

Cuando viene un estudiante asiático lo llevamos de la mano. Tienen un curso intensivo especial de español, tutorías y un mentor que los acompaña (E_S_SLT).

Los alumnos valoran de forma positiva la Oficina de Relaciones Internacionales que se encarga de su acogida.

Yo vine con una beca Santander. Mi país entra en crisis y no puedo usar ese financiamiento y entro en una crisis económica. La OCDS fue sumamente rápida y me ofrecieron en ese momento una solución. Fue difícil, pero yo sentí mucho apoyo de la ORI y de la gente del máster (GF2_E9).

Sin embargo, los docentes demandan más apoyo respecto al número de estudiantes internacionales, cómo realizar su acogida y, sobre todo, si tienen competencia lingüística y son conscientes de la política de la universidad.

\section{Discusión de los resultados y conclusiones}

Abordamos la investigación desde el enfoque de universidad intercultural inclusiva, que va más allá de la garantía de dar respuesta a las personas con discapacidad que acceden a la universidad. Se trata de una visión más amplia que no solo engloba al alumnado con necesidades de apoyo educativo, sino a cualquier estudiante sea cual sea su procedencia, género, identidad, estatus... No obstante, y a la vista de los resultados, este enfoque dista de ser asumido por las políticas universitarias que están más dirigidas a garantizar el acceso y permanencia de estudiantes con discapacidad.

Los responsables y técnicos de los servicios aseguran que se coordinan en función de las necesidades, pero que no existe una política que coordine esa relación, y los servicios se encuentran parcelados. Esto hace que se replantee la necesidad de una política más global para recoger ese enfoque intercultural inclusivo y abogar por acciones conjuntas, con el fin de asegurar el acceso y la permanencia de los estudiantes, independientemente de sus características personales, sociales o culturales.

En Latinoamérica encontramos algunos países como Perú o Ecuador (Pedroza y Villalobos, 2009; Fajardo, 2017) que también se centran en la 
discapacidad, y otros, como Venezuela y México, que promueven universidades para colectivos específicos desfavorecidos (Chiroleu, 2009). Sin embargo, nos interesa el planteamiento de países como Colombia, donde se considera a la educación superior como una institución diversa y heterogénea, que requiere de políticas para abarcar a todos los colectivos (Ministerio de Educación Nacional, 2013).

En la actualidad, en España ya hay iniciativas que proponen estrategias que inciden directamente en el desarrollo de políticas y prácticas inclusivas, como es el Proyecto de Formación Curricular en Diseño para Todas las Personas. Este tiene como objetivo principal introducir los conceptos básicos de Diseño para Todas las Personas y Accesibilidad Universal en los currículos de 15 titulaciones. No obstante, aún queda mucho camino por recorrer en ese aspecto.

Por otro lado, los resultados indican que la falta de la competencia lingüística es una barrera para el acceso y permanencia; ante ello se sugiere solicitar requisitos lingüísticos para los estudiantes que provienen de otras universidades. Este resultado no se aleja de otras investigaciones como un estudio de la Universidad de Alicante (Comes et al., 2014), donde los profesores consideran que los alumnos internacionales tienen poco conocimiento del castellano y del valenciano.

La UJI tiene una serie de mecanismos para fomentar la acreditación, formación y ayudas e incentivos que se basan en la Política Lingüística para la Internacionalización del Sistema Universitario Español (Bazo y González, 2017). Sin embargo, los participantes consideran que siguen siendo insuficientes y que es necesario crear más cursos para mejorar la competencia del español y acercar a los estudiantes al valenciano.

A grandes rasgos, podemos observar que la universidad tiene voluntad para suprimir las barreras y garantizar la accesibilidad a todas las personas. No obstante, la concepción de la accesibilidad sigue anclándose en una construcción pensada para personas con discapacidad y no en una universidad para todos. Así pues, es indispensable avanzar hacia el Diseño Universal (DU) que pretende diseñar y construir edificios y espacios que desde el inicio tomen en cuenta a la diversidad de la población y no solo las necesidades de las personas con discapacidad (Center for Universal Design, 2008).

Uno de los grandes retos en la construcción de una universidad más inclusiva es la formación del profesorado. En este sentido, es imprescindible fomentar un compromiso y actitud positiva hacia la diversidad (Fernández, 2012) y estar dispuesto a asumir la responsabilidad de buscar soluciones adecuadas para los estudiantes. La universidad tiene el desafío de diseñar 
entornos de aprendizaje que garanticen el éxito de sus alumnos, independientemente de sus características; ello supone desarrollar las competencias pedagógicas del profesorado.

En el contexto español, algunos estudios (Castellana y Sala, 2005; Moriña et al., 2015) ponen de manifiesto la falta de recursos en las aulas para favorecer la inclusión y la metodología poco adecuada del profesorado para asegurar la participación, el progreso y el éxito de los estudiantes.

Finalmente, es necesario destacar el trabajo de los servicios de apoyo, que, con un volumen muy alto de estudiantes y pocos recursos humanos, realizan una labor extraordinaria en la inclusión de los alumnos. Por ello, es esencial dignificar y reconocer a estos profesionales, pues hoy en día, de acuerdo con Alonso y Díez (2008), muchas universidades siguen sin tener claro cuáles son sus funciones, y los técnicos carecen de un perfil profesional específico.

\section{Referencias}

Aguado, Teresa et al. [coord.] (2006), Guia INTER. Una guia práctica para aplicar la educación intercultural en la escuela, España: Ministerio de Educación y Ciencia, Centro de Investigación y Documentación Educativa, Centro de Recursos y Planes de Atención a la Diversidad.

Bazo, Plácido y González, Dolores [coords.] (2017), Política Lingüistica para la Internacionalización del Sistema Universitario Español, España: Conferencia de Rectores de las Universidades Españolas.

Booth, Tony y Ainscow, Mel (2000), The Index for Inclusion: Developing Learning and Participation in Schools, Inglaterra: Centre for Studies on Inclusive Education.

Braun, Virginia y Clarke, Victoria (2006), "Using thematic analysis in psychology", en Qualitative Research in Psychology, vol.3, núm. 2. DOI: 10.1191/1478088706qp063oa Disponible en: www.tandfonline.com/doi/abs/10.1191/1478088706qp063oa [21 de julio de 2018].

Castellana, Montserrat y Sala, Ingrid (2005), "La universidad ante la diversidad en el aula", en Aula Abierta, núm. 85, España: Universidad de Oviedo.

Center for Universal Design (2008), Universal Design History. Disponible en: http://www. ncsu.edu/www/ncsu/design/sod5/cud/about_ud/udhistory.htm [19 de junio de 2019].

Cerón, Manuel (2006), Metodologias de la investigación social, Chile: LOM.

Chiroleu, Adriana (2009), "Políticas públicas de inclusión en la educación superior los casos de Argentina y Brasil”, en Pro-Posiçôes, vol. 20, núm. 2, Brasil: Universidade Estadual de Campinas.

Comes, Claudia et al. (2014), "Integración académica del alumnado universitario internacional de la UA: la perspectiva del profesorado", en Tortosa, M. et al. [coords.], XII Jornadas de Redes de Investigación en Docencia Universitaria. El reconocimiento docente: innovar e investigar con criterios de calidad, España: Universidad de Alicante.

Escudero, Juan Manuel (2006), "Compartir propósitos y responsabilidades para una mejora democrática de la educación”, en Revista de Educación, núm. 339. Disponible en: http://www.revistaeducacion.mec.es/re339/re339_03.pdf [01 de marzo de 2019]. 
European Commission (2017), Erasmus + Programme Guide, Bélgica: European Commission. Fajardo, M. Stella (2017), "La Educación Superior Inclusiva en Algunos Países de Latinoamérica: Avances, Obstáculos y Retos", en Revista Latinoamericana de Educación Inclusiva, vol. 11, núm. 1, Chile: Universidad Central de Chile.

Fernández, José María (2012), “Capacidades y competencias docentes para la inclusión del alumnado en la educación superior”, en Revista de la Educación Superior, vol. XLI, núm. 2, México: ANUIES.

Gibbs, Graham R. (2007), Analyzing qualitative data. Thousand Oaks, Estados Unidos: Sage.

Ministerio de Educación Nacional de Colombia (2013), Lineamientos Politica de Educación Superior Inclusiva. Disponible en: https://www.mineducacion.gov.co/1759/ articles-357277_recurso.pdf [01 de marzo de 2019].

Ministerio de Educación (2010), "Real Decreto 1791/2010, de 30 de diciembre, por el que se aprueba el Estatuto del Estudiante Universitario”, en Boletín Oficial del Estado, núm. 318, España: Gobierno de España.

Moliner, Odet et al. (2011), "Trazando procesos de cambio desde un modelo educativo intercultural inclusivo”, en Moliner, O. [ed.], Prácticas inclusivas: experiencias, proyectos y redes, España: Publicacions de la Universitat Jaume I.

Moriña, Anabel et al. (2015), "Faculty training: an unavoidable equirement for approaching more inclusive university classrooms", en Teaching in Higher Education, vol. 20, núm. 8, Reino Unido: Penny Jane Burke.

Pedroza, René y Villalobos, Guadalupe (2009), Politicas compensatorias para la equidad en la educación superior en Ecuador y Perú, Argentina: Centro Argentino de Estudios Internacionales.

Pérez, Gloria (1994), Investigación cualitativa I: Retos e interrogantes: métodos, España: La Muralla.

Rifa, Montserrat et al. [eds.] (2014), "Nuevos desafíos para la inclusión social y la equidad en instituciones de educación superior”, en Actas del III Congreso Internacional MISEAL, España: Universitat Autónoma de Barcelona.

Sales, Auxiliadora et al. (2012), "Escuela intercultural inclusiva: estudio de caso sobre procesos de autoevaluación”, en Revista de Educación, núm. 358, España: Ministerio de Educación, Cultura y Deporte.

Sales, Auxiliadora y García, Rafaela (1997), Programas de Educación Intercultural, España: Desclée De Brouwer.

Sales, Auxiliadora et al. (2010), La construcción de la escuela intercultural inclusiva desde procesos de investigación-acción, España: Universitat Jaume I.

Simons, Helen (2011), El estudio de caso: Teoría y práctica, España: Morata.

Stake, Robert (1998), Investigación con estudio de casos, España: Morata.

Sverdlick, Ingrid et al. (2005), "Desigualdad e inclusión en la educación superior", en Un estudio comparado en cinco paises de América Latina, Argentina: Laboratorio de Políticas Públicas de Buenos Aires.

Unesco (2015), Declaración de Incheon, Educación 2030. Hacia una educación inclusiva y equitativa de calidad y un aprendizaje a lo largo de la vida para todos, Francia: Unesco.

Unesco (2017), Guía para asegurar la inclusión y la equidad en la educación. Disponible en:http://unesdoc.unesco.org/images/0025/002595/259592s.pdf [01 de marzo de 2019]. 


\section{Anexo}

\section{Figura 1}

\section{Elementos políticas inclusivas}

NORMATIVAS, PLANES Y POLÍTICAS

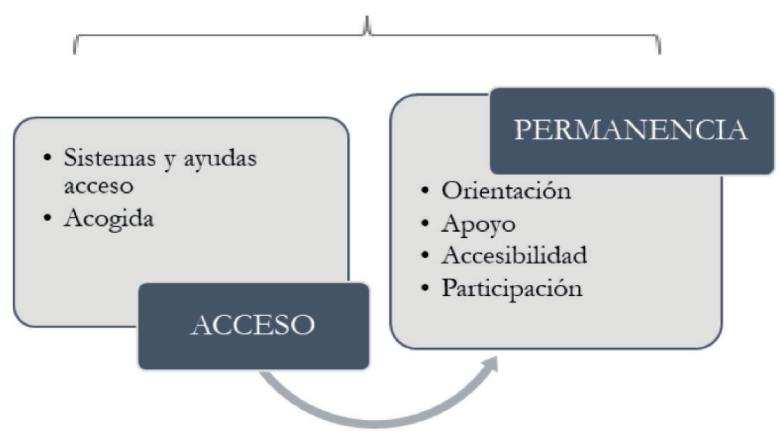

Fuente: Elaboración propia. 


\section{Tabla 1}

\section{Servicios relacionados con la atención a la diversidad}

UNIDAD DE
APOYO
EDUCATIVO (USE)

Este servicio se divide en diferentes ámbitos de trabajo:

- Unidad de Orientación Preuniversitaria (UOP) ofrece orientación para favorecer la transición a la universidad y fomentar la coordinación con los orientadores de los centros educativos.

- Unidad de Orientación Universitaria (UOU) es el área encargada de asesorar al estudiantado en su etapa universitaria, así como ofrecerle recursos para mejorar y hacer crecer sus potencialidades.

- Unidad de Diversidad y Discapacidad (UDD) se encarga de gestionar el Programa de Atención a la Diversidad que trata de dar apoyo la comunidad universitaria que presentan diversidad funcional y/o necesidades educativas especiales.

- Unidad de Formación e Innovación Educativa (UFIE) dentro de sus ámbitos de actuación encontramos la formación del profesorado, la innovación educativa y la certificación de méritos.
Unidad que centraliza, coordina y gestiona los asuntos, procedimientos y trámites administrativos del alumnado matriculado en estudios oficiales de la universidad. Se encarga de la preinscripción, matrícula, convalidaciones, becas y títulos. (SGDE)

UNIDAD DE IGUALDAD (UI)
Se encarga de desarrollar el Plan de igualdad de la UJI, velar por el cumplimiento de la legislación en igualdad y promover la implantación de la transversalidad de género en todas las políticas universitarias.

SERVICIO DE

LENGUAS Y TERMINOLOGÍA (SLT)
Se encarga de organizar y resolver las necesidades sobre las lenguas y los modelos lingüísticos que tiene utilizan la universidad en todos sus ámbitos de actuación. 


\begin{tabular}{|c|c|}
\hline $\begin{array}{l}\text { OFICINA DE } \\
\text { RELACIONES } \\
\text { INTERNACIONALES } \\
\text { (ORI) }\end{array}$ & $\begin{array}{l}\text { Se ocupa de difundir y gestionar los programas nacionales e } \\
\text { internacionales como Erasmus+, SICUE, y otros propios que } \\
\text { promueven el intercambio de estudiantes, PDI y PAS. Una } \\
\text { de las tareas más importantes es el apoyo a estudiantes de in- } \\
\text { tercambio en el registro, matrícula, alojamiento a través de la } \\
\text { orientación. }\end{array}$ \\
\hline $\begin{array}{l}\text { OFICINA } \\
\text { COOPERACIÓN } \\
\text { AL DESARROLLO } \\
\text { Y SOLIDARIDAD } \\
\text { (OCDS) }\end{array}$ & $\begin{array}{l}\text { Tiene como misión difundir y desarrollar los principios de so- } \\
\text { lidaridad y cooperación entre la comunidad universitaria. Esta } \\
\text { oficina gestiona el voluntariado, una parte muy importante en } \\
\text { la atención a la diversidad. }\end{array}$ \\
\hline $\begin{array}{l}\text { OFICINA DE } \\
\text { PREVENCIÓN } \\
\text { Y GESTIÓN } \\
\text { MEDIOAMBIENTAL } \\
\text { (OPGM) }\end{array}$ & $\begin{array}{l}\text { Dentro de esta oficina encontramos el Centro de Salud que } \\
\text { proporciona asistencia sanitaria a la comunidad universitaria. } \\
\text { Este servicio se encarga de las adaptaciones del espacio de } \\
\text { estudio o del trabajo. Según las necesidades de la persona so- } \\
\text { licitante (ya sea estudiante, PDI o PAS) el centro realiza una } \\
\text { valoración individualizada de las características personales y } \\
\text { del entorno de trabajo o estudio. }\end{array}$ \\
\hline SALUSEX & $\begin{array}{l}\text { Es la Unidad de Investigación sobre Sexualidad y SIDA que } \\
\text { servicios de prevención y tratamiento de problemas asociados } \\
\text { a la sexualidad y el SIDA. Se trata de un centro sanitario, pero } \\
\text { no depende de ningún vicerrectorado. El grupo también realiza } \\
\text { acciones de formación y sensibilización. }\end{array}$ \\
\hline
\end{tabular}

Fuente: Elaboración propia. 
Tabla 2

Participantes en el estudio

\section{PARTICIPANTES}

\section{1 técnicos/}

directores Servicios: USE, ORI, OCDS, UI, OPGM, SLT, Salusex, CENT y SASC académicos

Departamentos: Ingeniería y Ciencias de los Computadores, Estudios 8 docentes Ingleses, Derecho Público, Sociología, Ingeniería de Sistemas Industriales y Diseño, y Ciencias de la Comunicación

13 estudiantes

Diversas nacionalidades, de grado y máster, relación con los servicios, con y sin ayuda de becas.

7

Ordenación académica y profesorado; Internacionalización, vicerrectores

Cooperación y multilingüismo; Planificación Estratégica, Calidad e Igualdad; Investigación y Doctorado; Campus, Infraestructuras y Nuevas Tecnologías; Estudios y Estudiantes, Empleo e Innovación

Rector

Fuente: Elaboración propia. 


\section{Tabla 3}

\section{Documentos institucionales analizados}

\begin{tabular}{|c|c|}
\hline Estatutos UJI & $\begin{array}{l}\text { Información de la web Programa Atención } \\
\text { Diversidad }\end{array}$ \\
\hline Modelo educativo 2016 & Plan de promoción de la investigación 2017 \\
\hline Informe Modelo Educativo 2017 & Carta Servicio Actividades Socioculturales \\
\hline Código Ético Universidad 2018 & Descripción Asociaciones \\
\hline Ilustraciones Código Ético & Reglamento registro asociaciones \\
\hline Plan Estratégico 2018 & Tipos de becas y ayudas estudiantes \\
\hline Plan Igualdad 2016-2020 & Normativa Acceso Estudiantes +25 \\
\hline $\begin{array}{l}\text { Plan plurianual de Multilingüismo } \\
\text { 2016-2018 }\end{array}$ & Normativa Acceso Estudiantes +45 \\
\hline Política Lingüística & Normativa Acceso Estudios Parciales \\
\hline Manual Usos Lingüísticos & Normativa Académica Estudios de Grado \\
\hline Manual Usos Lingüísticos Ilustraciones & Normativa Académica Estudios de Máster \\
\hline $\begin{array}{l}\text { Informe Anual Plan Multilingüismo } \\
2017\end{array}$ & Normativa Compensación Curricular \\
\hline Guía para la Docencia Multilingüe & Normativa Evaluación \\
\hline Plan Internacionalización & Normativa Permanencia \\
\hline $\begin{array}{l}\text { Plan de Atención a la Diversidad para } \\
\text { estudiantes }\end{array}$ & Guía Voluntariado UJI \\
\hline $\begin{array}{l}\text { Plan de Atención a la Diversidad para } \\
\text { PDI con discapacidad }\end{array}$ & Reglamento Consejo Estudiantes \\
\hline Guía Atención a la Diversidad para PDI & $\begin{array}{l}\text { Estatuto del Estudiantes Universitario } \\
\text { (BOE) }\end{array}$ \\
\hline
\end{tabular}

Protocolo para la detección, prevención y actuación en los supuestos de acoso laboral, acoso sexual, acoso por razón de sexo, acoso por orientación sexual e identidad y expresión de género

Fuente: Elaboración propia. 
Tabla 4

\section{Codificación}

\begin{tabular}{|c|c|c|}
\hline \multicolumn{3}{|r|}{ INSTRUMENTOS } \\
\hline ENTREVISTA & \multicolumn{2}{|l|}{$\mathrm{E}$} \\
\hline GRUPO FOCAL & \multicolumn{2}{|l|}{ GF } \\
\hline $\begin{array}{l}\text { ANÁLISIS } \\
\text { DOCUMENTAL }\end{array}$ & \multicolumn{2}{|c|}{$\mathrm{AD}$} \\
\hline \multicolumn{3}{|l|}{ PARTICIPANTES } \\
\hline \multirow{12}{*}{ SERVICIOS } & \multirow{12}{*}{$S$} & USE (Unidad Apoyo Educativo) \\
\hline & & UDD (Unidad Discapacidad y Diversidad) \\
\hline & & UFIE (Unidad de Formación e Innovación Educativa) \\
\hline & & UI (Unidad de Igualdad) \\
\hline & & UARE (Unidad de Atención y Representación de Estudiantes) \\
\hline & & ORI (Oficina Relaciones Internacionales) \\
\hline & & OCDS (Oficina de Cooperación Al Desarrollo) \\
\hline & & OPGM (Oficina de Prevención I Gestión Medioambiental) \\
\hline & & SLT (Servicio de Lenguas Y Terminología) \\
\hline & & SALUSEX \\
\hline & & CENT (Centro de Educación y Nuevas Tecnologías) \\
\hline & & SASC (Servicio de Actividades Socioculturales) \\
\hline ESTUDIANTES & $\mathrm{E}$ & \\
\hline DOCENTES & $\mathrm{D}$ & \\
\hline VICERECTORES & VR & \\
\hline RECTOR & $\mathrm{R}$ & \\
\hline
\end{tabular}

Fuente: Elaboración propia. 


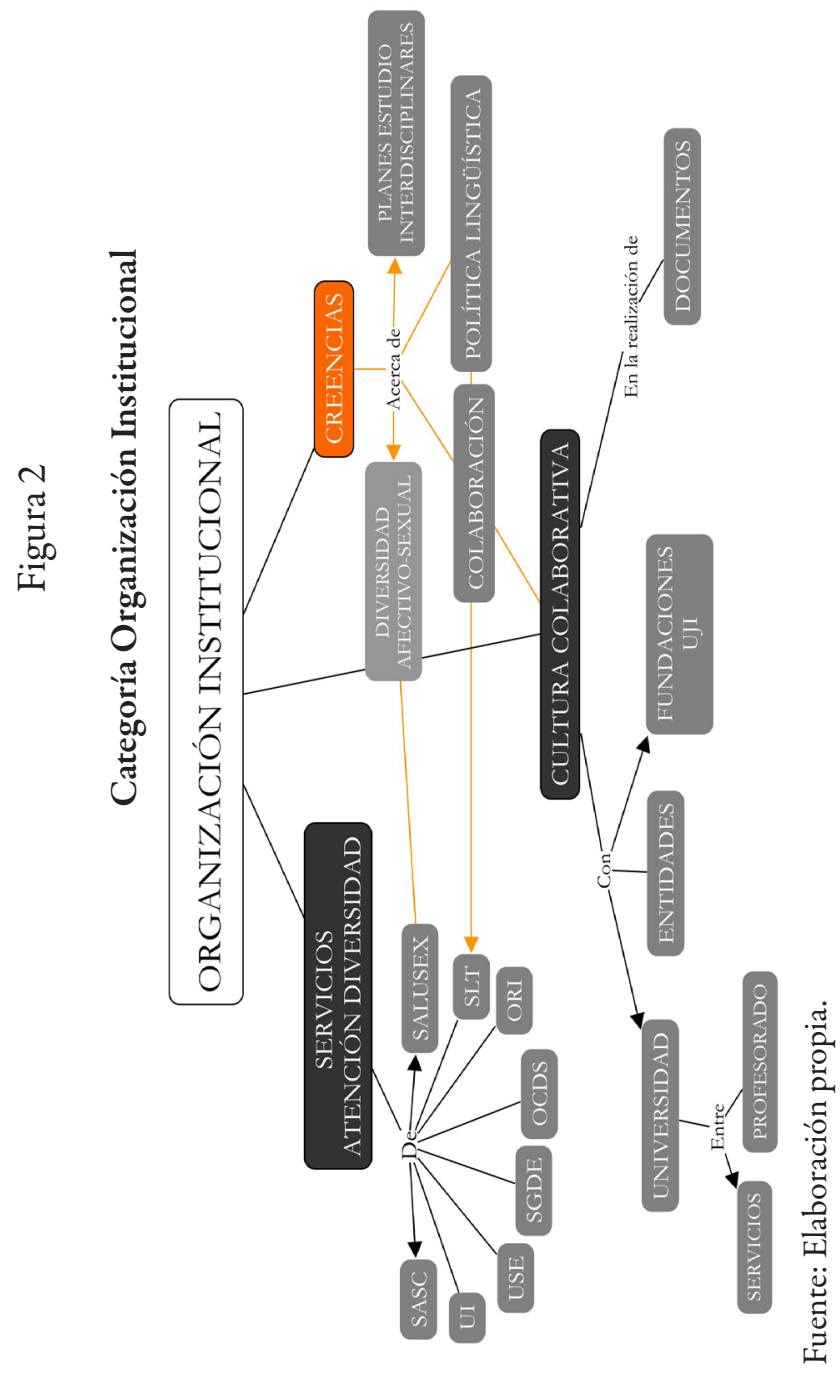


Alicia Benet-Gil

Desarrollo de politicas inclusivas en la educación superior

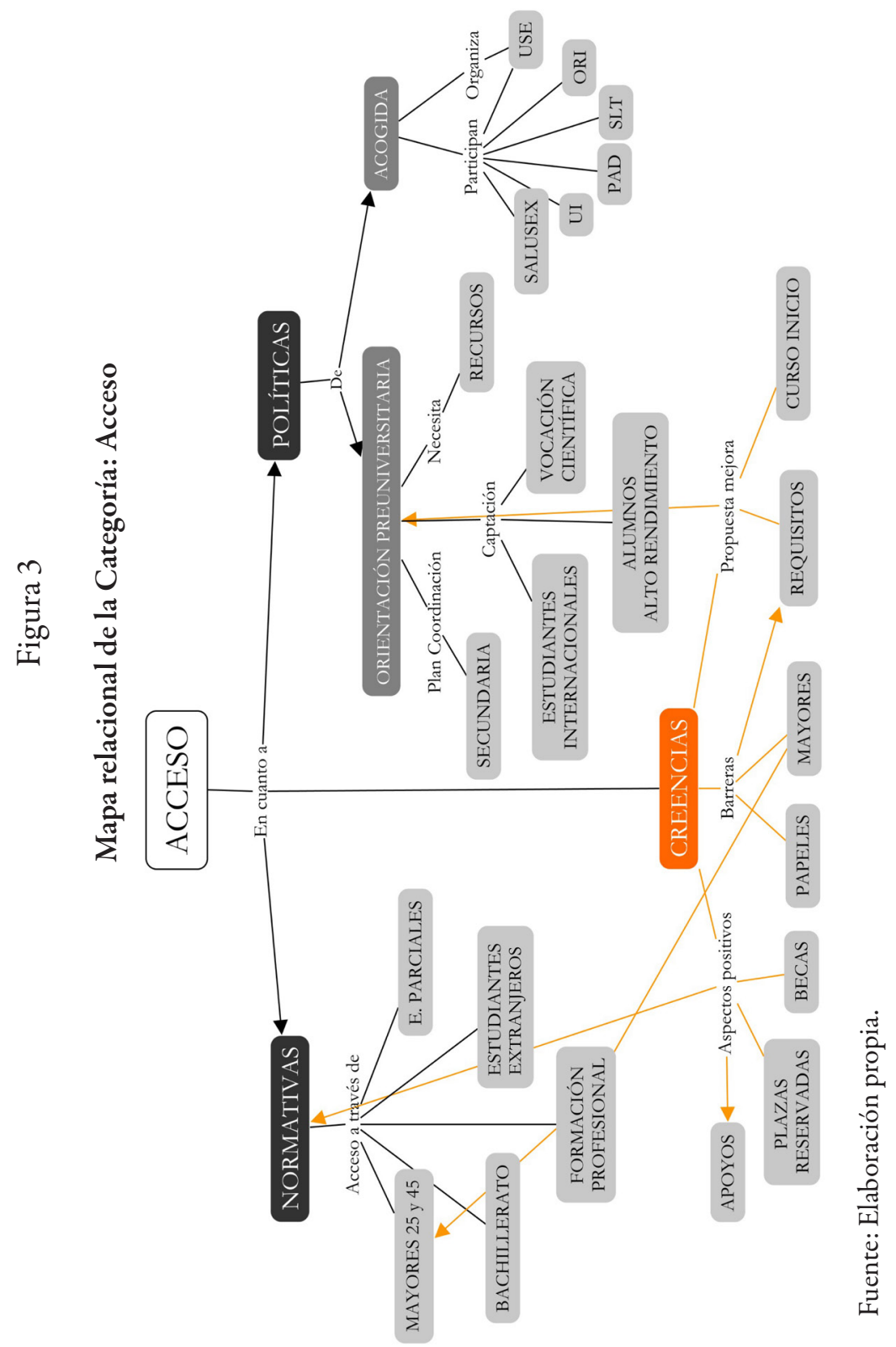




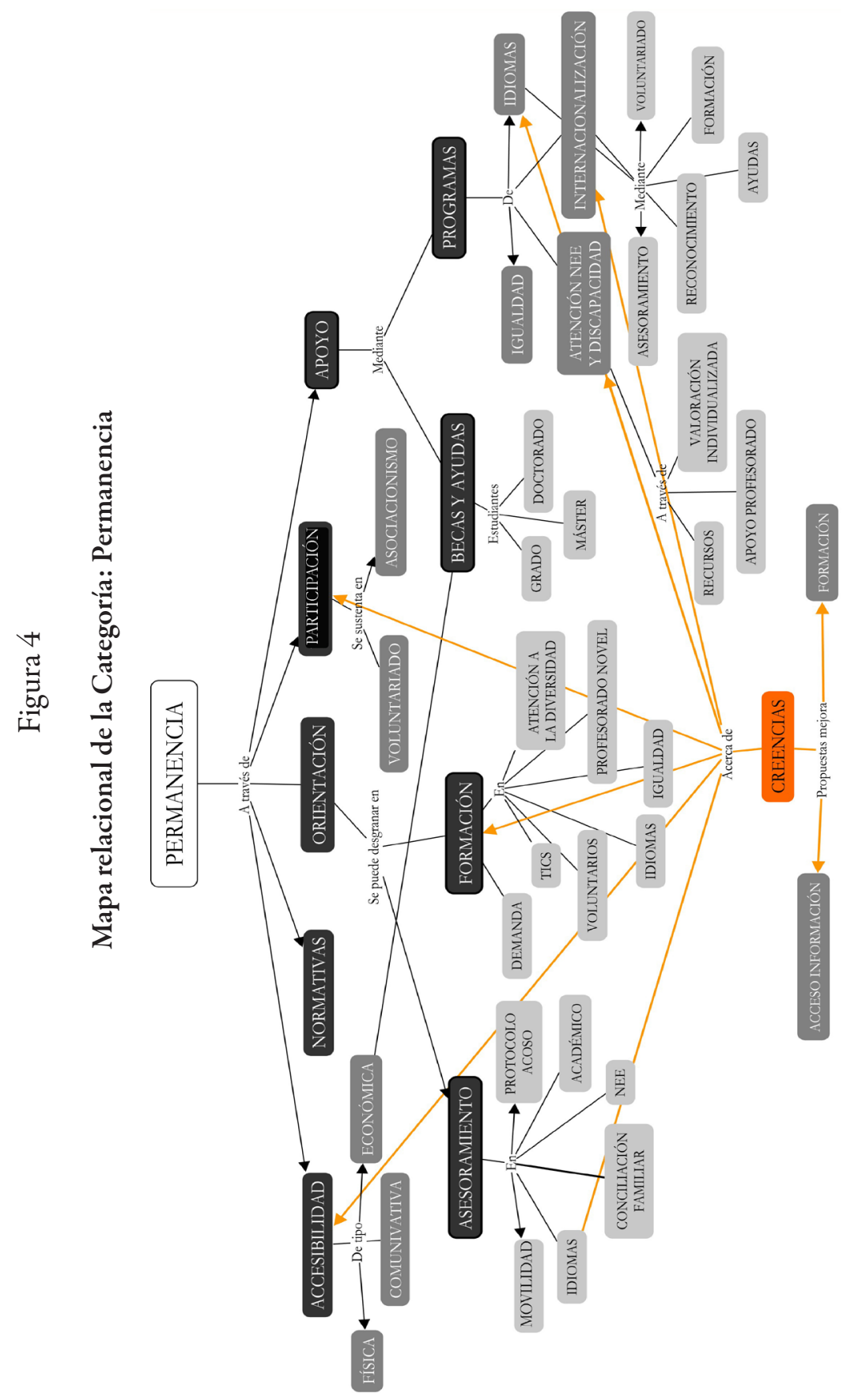

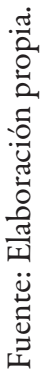


Alicia Benet-Gil. Profesora departamento de Pedagogía y Didáctica de les Ciencias Sociales, la Lengua y la Literatura. Líneas de investigación: educación intercultural inclusiva en la educación superior, Perspectivas sociocomunitarias en educación y escuela incluida, formación docente e innovación metodológica. Publicaciones recientes: Moliner, O., Yazzo, M. A., y Benet, A. (2018), "Educación superior intercultural inclusiva. Experiencias de investigación en la Universitat Jaume I (España) y la Fundación Universitaria Los Libertadores (Colombia)", en Assurer la réussite de tous les élèves. Perspectives internationales. Reims (França), Francia: Éditions et Presses Universitaires de Reims; Molina, M., Benet, A., y Doménech, A. (2019), "La tutoría entre iguales: Un elemento clave en las aulas interculturales inclusivas", en Revista Complutense de Educación, núm. 1, vol. 30, España; Traver, J. A., Sales, A., Moliner, O., Sanahuja, A. y Benet, A. (2018), "Hacia una escuela incluida en su territorio: Análisis de una práctica comunitaria", en Edetania, núm. 53, España. 